\title{
COLUMN SEQUENCES IN HAUSDORFF MATRICES
}

\section{J. D. BUCKHOLTZ}

Corresponding to each sequence $d$ of complex numbers, the Hausdorff matrix $H=H(d)$ is given by

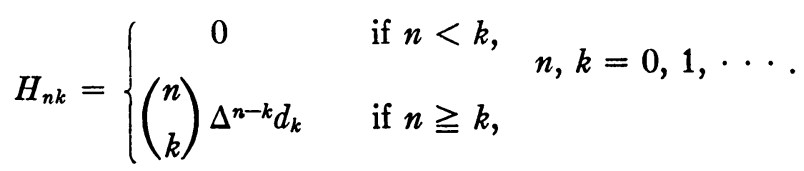

For convenience, we shall denote the $k$ th column sequence by $h^{(k)}$, i.e., $h_{n}^{(k)}=H_{n k}, n=0,1, \cdots$. For each $k \geqq 1, C^{k}$ will denote the $k$ th power of the Cesàro matrix $(C, 1)$. We shall make use of the fact that, regarded as summability methods, $C^{k}$ and $(C, k)$ are equivalent $[1, \mathrm{p} .103]$. If the $C^{k}$ transform (and consequently the $(C, k)$ transform) of a sequence $s$ has limit $x$, we shall abbreviate this by $s_{n} \rightarrow$ $x(C, k)$.

For Hausdorff matrices which satisfy the condition

$$
\sum_{k=0}^{n}\left|B_{n k}\right| \leqq M \quad(M \text { independent of } n),
$$

it is well known $[1$, p. 255$]$ that $h^{(0)}$ converges and that every other column sequence converges to zero. The purpose of this note is to obtain a weaker form of this result for all Hausdorff matrices for which $h^{(0)}$ converges.

Theorem. If $H$ is a Hausdorff matrix and $h^{(0)}$ converges, then $h_{n}^{(k)} \rightarrow 0(C, k)$ for every positive integer $k$.

Proof. The proof depends mainly on the sequence identity

$$
C h^{(k)}=C h^{(k-1)}-\frac{1}{k} h^{(k-1)},
$$

where $C h^{(k)}$ denotes the $C$ transform of the sequence $h^{(k)}$. Noting that, for $n \geqq k$, the $n$th term of $C h^{(k)}$ is

$$
\frac{1}{n+1} \sum_{p=k}^{n}\left(\begin{array}{l}
p \\
k
\end{array}\right) \Delta^{p-k} d_{k}
$$

a verification of (1) follows from the identities

$$
\Delta^{p-k} d_{k}=\Delta^{p-k} d_{k-1}-\Delta^{p-k+1} d_{k-1} \text { and }\left(\begin{array}{l}
p \\
k
\end{array}\right)-\left(\begin{array}{c}
p-1 \\
k
\end{array}\right)=\left(\begin{array}{c}
p-1 \\
k-1
\end{array}\right) \text {. }
$$

Presented to the Society, July 5, 1962; received by the editors July 22, 1962. 
If in (1), $k=1$, convergence of $h^{(0)}$ immediately implies $h_{n}^{(1)} \rightarrow 0$ $(C, 1)$. Suppose now that $k-1$ is a positive integer for which $h_{n}^{(k-1)}$ $\rightarrow 0(C, k-1)$. Applying the $C^{k-1}$ matrix to both sides of (1), we have

$$
C^{k} h^{(k)}=C^{k} h^{(k-1)}-\frac{1}{k} C^{k-1} h^{(k-1)} .
$$

Since $C^{k-1} h^{(k-1)}$ has limit zero, so does $C^{k} h^{(k-1)}$, and therefore $C^{k} h^{(k)}$ has limit zero. This completes the proof.

CoROLlaRY. If $H$ is a Hausdorff matrix and $h^{(0)}$ converges, then for every positive integer $k$ for which $h^{(k)}$ converges, $h^{(k)}$ has limit zero.

Proof. $(C, k)$ summability is regular.

\section{REFERENCE}

1. G. H. Hardy, Divergent series, Clarendon, Oxford, 1949.

University of North Carolina 